La plupart des auteurs sont, d'une part presque tous partisans de l'emploi de la filtration-centrifuge, de l'homogénéisation et du vide; ces différents traitements sont connus et nous ne décrirons pas les appareils que nécessite leur utilisation.

Le froid, préconisé par certains spécialistes n'est en général indiqué que comme mode de conservation pendant un temps très limité pratiquement, et nous n'insisterons pas sur ce procédé. C'est donc surtout l'emploi de la chaleur qui retiendra désormais notre attention :

Utilisée avec la filtration-centrifuge, l'homogénéisation et au besoin le vide sur des laits recueillis aussi proprement que possible et préparés dans un miminum de temps après la traite, la chaleur nécessaire à une stérilisation pratique permet d'obtenir un produit dont ni le goût, ni la couleur, ni les principaux éléments ne sont considérablement altérés au point de vue de la digestibilité. La vitamine C est évidemment fortement touchée, surtout en présence d'oxygène, et c'est là, avec l'insolubilisation de l'acide phosphorique et des sels de chaux, les principaux inconvénients d'un lait stérilisé destiné à l'alimentation de nourrissons, mais ils ne sont pas prohibitifs.

(à suivre)

\title{
LE CANCER CHEZ LES FEMELLES DANS SES RELATIONS AVEC LA MAMELLE ET LE TRACTUS URO-GÉNITAL
}

\author{
par M. Ch. LOMBARD.
}

Chef des travaux d'Anatomie Pathologique à l'Ecole Vétérinaire de Lyon.

L'étude du cancer sous quelque angle qu'on l'envisage est toujours pleine d'un merveilleux attrait. Et ce n'est pourtant pas - encore que la Cancérologie comparée ne date guère que du $\mathrm{XIX}^{\mathrm{e}}$ siècle - que d'innombrables chercheurs ne se soient efforcés de résoudre le problème de son étiologie, de sa pathogénie, de sa thérapeutique.

Suivant la définition de Roussy et WoLf, il faut entendre sous le terme précis de cancer, seules "les néoformations qui n'ont pas la structure des processus inflammatoires ou parasitaires connus, qui ont tendance à persister et à s'accroître, qui sont douées de propriétés envahissantes et destructives, qui font des métastases, qui récidivent après ablation, qui tuent par eachexie, et qui sont inoculables en série dans une même espèce animale. "Ainsi le cancérologue doit toujours avoir en vue non seulement les tumeurs malignes spontanées, mais encore les néoplasies expérimentales. C'est, dans cet esprit, que nous exposerons nos connaissances actuelles sur le cancer chez les femelles dans ses relations avec la mamelle et le tractus uro-génital.

$$
\text { *** } *
$$

Si tous les types histologiques des tumeurs humaines ont été, d'une manière générale, observés sur les animaux, leur fréquence est fort variable suivant les diverses espèces. Tous les auteurs reconnaissent que les carnivores paient indiscutablement le plus lourd tribut aux 
tumeurs malignes. Viennent ensuite, par ordre de fréquence, cheval, bœuf, âne, mulet, pore, mouton et chèvre pour ce qui est des animaux domestiques. La courbe de fréquence du cancer pour chaque individu, compte tenu de son âge de maturité, reste d'ailleurs très sensiblement parallèle à celle de l'homme.

Il est de notion courante que le cancer prédomine chez les femelles domestiques. Rien n'est moins prouvé, si ce n'est chez les carnivores. Chez les solipèdes, en effet, le seul document précis que nous possédions (statistique de Césari) met, en évidence, la plus grande fréquence du eancer des mâles. A ce point de vue du sexe, on s'est demandé si la gestation pouvait avoir quelque influence sur le développement du cancer. Mais les observations, recueillies sur la souris et la lapine surtout, ne permettent guère de conclure. Tout ce que l'on peut affirmer, c'est la compatibilité du cancer utérin avec la gestation.

A noter que le tractus uro-génital semble particulièrement prédisposé au cancer. U. LEBLANC, CADiot, les premiers, signalent la fréquence insolite du cancer mammaire chez les femelles des carnivores. Pour STICKER même, $45 \%$ des cas d'épithéliomas appartiendraient à la mamelle. En matière de sarcomes, V. BALt met sur la même courbe de fréquence la peau et les mamelles. Lrínaux pense que, chez les bovidés, les mamelles, l'utérus et le rein présentent la plus forte proportion de cancers, et Ć́SARI, qui ne s'est occupé que des épithéliomas des équidés seulement, indique, chez la jument, la mamelle, puis le rein comme étant les organes le plus souvent lésés.

Statistique personnelle établissant la fréquence par appareil

$1^{0}$ Des tumeurs bénignes et malignes.

Appareil digestif.

$\begin{array}{rrrrrrcc}\text { Chien } & \text { Chienre } & \text { Chat } & \text { Chatte } \text { Hlev. Jum. Beuf } & \text { Vache } \\ 22 & 8 & 10 & 2 & \text { " } & \text {, } & 1 & 3 \\ 2 & 21 & \text { " } & 8 & 1 & 2 & \text { " } & 1 \\ 9 & n & \text { " } & \text { " } & \text { " } & \text { " } & \text { " } & \text { " } \\ 7 & 1 & 3 & \text { " } & \text { " } & \text { " } & \text { " } & \text { " } \\ 5 & 2 & 3 & 1 & \text { " } & \text { " } & \text { " } & \text { " } \\ 3 & 2 & 4 & 1 & 1 & \text { " } & \text { " } & 1 \\ 1 & \text { " } & 1 & \text { " } & 2 & 1 & \text { " } & \text { " } \\ 3 & n & 1 & \text { " } & 2 & \text { " } & \text { " } & 2 \\ 7 & 2 & \text { " } & \text { " } & 1 & \text { " } & \text { " } & \text { " } \\ 1 & \text { " } & 2 & \text { " } & \text { " } & \text { " } & \text { " } & \text { " }\end{array}$

$2^{\circ}$ Des tumeurs malignes.

\begin{tabular}{|c|c|c|c|c|c|c|c|}
\hline Appareil génital.............. & 1 & 7 & $n$ & 4 & 1 & 1 & $n$ \\
\hline Sang. . . . . . . . . . . . . . . & 7 & 1 & 3 & " & " & 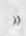 & \\
\hline Appareil digestif. . . . . . . . . . . . & 2 & 3 & & & $n$ & & \\
\hline Squelette $\ldots \ldots \ldots \ldots \ldots \ldots \ldots$ & \pm & $p$ & $n$ & & $n$ & (1) & \\
\hline Appareil respiratoire $\ldots \ldots \ldots \ldots \ldots$ & 2 & 1 & & & 1 & & \\
\hline Appareil urinaire.............. & " & $\eta$ & & & 2 & 1 & \\
\hline Peau $\ldots \ldots \ldots \ldots \ldots \ldots \ldots \ldots$ & 2 & " & $n$ & & n & "1" & \\
\hline Corps thyroïdes . . . . . . . . . . . . & 4 & 1 & " & & n & & \\
\hline Capsules surrénales ... . . . . . . . . & $n$ & 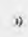 & » & D) & $n$ & 1" & \\
\hline
\end{tabular}


Il faut bien reconnaître, du reste, que c'est surtout chez les carnivores, canidés, en particulier, que le cancer mammaire, quelle que soit sa forme histologique, est extrêmement banal. Macroscopiquement il peut ressembler au cancer en plastron de la femme (Liénaux). Chez la jument et la vache, il est beaucoup plus rare. Sur la souris, l'adéno-épithélioma est le type de tumeur le plus commun.

Le sarcome et l'épithéliome du rein, fréquents chez les solipèdes, se voient aussi sur les carnivores et les suidés.

Le eancer de la vessie, signalé chez les herbivores et les carnivores par G. PETit, est assez rare dans toutes les espèces.

Quant au cancer de l'ovaire, tout comme ceux, plus rares, de l'utérus et du vagin, il est connu dans toutes les espèces, ceux-ci se rencontrant surtout chez la vache.

Chose curieuse, les deux seuls cas de chorio-placentome, connus en pathologie comparée, ont été recueillis sur la vache (FAURE). Les suidés ont surtout des sareomes du rein et de l'utérus. La chienne présente des kystes prolifères de l'ovaire comparables à ceux de la femme (G. PeтrT). On connaît, en ce qui concerne la lapine, l'épithélioma du rein, de la mamelle, de l'utérus. La souris présente, le plus souvent, des tumeurs épithéliales, du type pavimenteux ou glandulaire, développées aux dépens des glandes mammaires.

$$
\text { *** }
$$

La notion importante qui domine tous ees faits, c'est qu'en pathologie comparée tout comme le Professeur DeLbet l'a si bien souligné en médecine humaine, les organes concourant à la reproduction et fonctionnant d'une manière intermittente : mamelles, ovaires... semblent particulièrement prédisposés au cancer. La remarque est si générale qu'elle s'applique même aux amphibies. Et dès lors, s'il y avait un rapport entre la fonction des organes et la fréquence de leurs cancers, la question qui se poserait ne serait-elle pas : "Est-ce parce qu'ils fonctionnent trop ou parce qu'ils ne fonctionnent pas assez?" (DELBET).

$$
\text { ** * }
$$

Soulignons que ces néoplasmes du tractus uro-génital semblent entretenir parfois de curieux rapports avec l'inflammation. Dans quatre cas de tumeurs vaginales que nous avons recueillies sur des vaches, qui avaient accouché quelque temps auparavant, les froissements et déchirures vaginaux paraissaient avoir contribué à accélérer le développement de ces tumeurs. Expérimentalement, Teutschlannder produit même le cancer utérin chez un rat femelle en combinant les injections intra-vaginales de goudron avec le badigeon des parties extérieures environnantes.

Mais les faits sont encore plus nets au niveau de la mamelle. Tout se passe, en effet, comme si la mammite précédait souvent le cancer. 
Ainsi la mamelle est le siège de prédilection des tumeurs, surtout chez les vieilles chiennes, dont les mamelons volumineux et flétris témoignent de leurs nombreuses portées. Chez les souris également, le cancer spontané mammaire apparaît plus souvent aux mamelles particulièrement tétées, lorsque ces animaux ont eu des portées répétées. Du reste, par l'injection irritante intra-mammaire de goudron chez la souris, YAMAGIWA et Murayama, Seedorff obtiennent du cancer.

$\mathrm{Il}$ est rare d'ailleurs que le trauma intervienne seul : il ne fait, le plus souvent, que favoriser l'entrée en scène des agents toxi-infectieux. Quant aux mammites purement toxi-infectieuses, observées surtout chez la vache, elles sont rarement associées aux tumeurs.

\section{$*^{*} *$}

Ces quelques exemples - si aucune objection ne s'élevait contre eux - militeraient en faveur de l'influence des agents irritants dans la pathogénie du cancer. Mais il faut bien remarquer que la mamelle de la vache soumise à un travail intensif, et très exposée aux inflammations, est rarement cancéreuse. Aussi Peller a-t-il pu écrire, à propos de la femme - et la remarque est vraie pour la vache - que l'allaitement et la mastite ne peuvent être considérés comme favorisant la production du cancer.

Ainsi apparaît à l'évidence que le cancer ne se greffe point systématiquement sur les lésions inflammatoires. Les conditions nécessaires à son développement sont si complexes que, quel que soit l'agent irritant employé, l'expérimentateur ne réussit point à produire du cancer à coup sûr. Parfois a-t-il, comme CADIOT, un échec total. "Sur plusieurs chiennes vieilles et eczémateuses, nous avons pratiqué des irritations mécaniques des mamelles. Ces glandes ont été, tous les jours ou tous les deux jours, comprimées et contusionnées au moyen d'une forte pince en bois. Bien que les expériences aient été prolongées pendant des mois, jamais nous n'avons obtenu de néoplasmes. ") (САDIOT).

Il n'en est pas moins vrai, suivant la remarque de Forgue et JEANBRAE, que l'action des agents phlogogènes est indiscutable toutes les fois que la lésion inflammatoire, suffisamment importante, porte sur une région indemne auparavant et qu'il y a correspondance entre la lésion et la tumeur subséquente, la date d'apparition de la tumeur rendant la filiation vraisemblable et la continuité des manifestations pathologiques étant parfaite depuis l'accident.

Dans ces cas, on voit d'ordinaire se greffer sur la lésion inflammatoire un ou plusieurs adénomes, qui peuvent subir ultérieurement l'involution épithéliomateuse. Ainsi s'explique le stade transitoire de l'adénoépithéliome, pas rare dans la mamelle enflammée des carnivores. 